\title{
Conversion of gas into stars in the Galactic center
}

\author{
S. N. Longmore \\ Astrophysics Research Institute, Liverpool John Moores University, \\ IC2, Liverpool Science Park 146 Brownlow Hill, Liverpool L3 5RF United Kingdom. \\ email: s.n.longmore@ljmu.ac.uk
}

\begin{abstract}
The star formation rate in the central $500 \mathrm{pc}$ of the Milky Way is lower by a factor of $>10$ than expected for the substantial amount of dense gas it contains, which challenges current star formation theories. I discuss which physical mechanisms could be causing this observation and put forward a self-consistent cycle of star formation in the Galactic center, in which the plausible star formation inhibitors are combined. Their ubiquity suggests that the perception of a lowered central SFR should be a common phenomenon in other galaxies with direct implications for galactic star formation and also potentially supermassive black hole growth. I then describe a scenario to explain the presence of super star clusters in the Galactic center environment, in which their formation is triggered by gas streams passing close to the minimum of the global Galactic gravitational potential at the location of the central supermassive black hole, Sgr A* . If this triggering mechanism can be verified, we can use the known time interval since closest approach to Sgr A* to study the physics of stellar mass assembly in an extreme environment as a function of absolute time. I outline the first results from detailed numerical simulations testing this scenario. Finally, I describe a study showing that in terms of the baryonic composition, kinematics, and densities, the gas in the Galactic center is indistinguishable from high-redshift clouds and galaxies. As such, the Galactic center clouds may be used as a template to understand the evolution (and possibly the life cycle) of high-redshift clouds and galaxies.
\end{abstract}

Keywords. Galaxy: center — star: formation — ISM: kinematics and dynamics

\section{Introduction}

The process of converting gas into stars underpins much of astrophysics and cosmology. An end-to-end understanding of this process as a function of environment is therefore of fundamental importance to our understanding of the Universe. Over the last few decades the theoretical picture describing the formation of individual stellar systems has matured, underpinned by observations of the closest star formation regions, which lie at typical distances of a few hundred parsecs. Over a similar time frame, observations comparing the distribution of gas and young stars within galaxies have developed our understanding of how star formation proceeds on kpc scales (e.g. Schmidt 1959, Elmegreen 1987, Silk 1997, Kennicutt 1998a, Kennicutt 1998b, Bigiel et al. 2008, Leroy et al. 2008).

Recently, attempts have been made to link our understanding of star formation across this large range of spatial scales in a unified picture of the star formation process (e.g. Lada et al. 2012, Krumholz, Dekel \& McKee 2012). Several studies find evidence for an empirical gas surface density threshold in nearby molecular clouds of $\sim 120 \mathrm{M}_{\odot} / \mathrm{pc}^{2}$, above which gas is efficiently converted into stars (Lada et al. 2010, Heiderman et al. $2010) \dagger$. They convert this surface density threshold to a critical volume density of

$\dagger$ Although note that Gutermuth et al. (2011) and Burkert \& Hartman (2013) come to a different conclusion. 
$\sim 10^{4} \mathrm{~cm}^{-3}$ that gas must reach before efficient star formation can commence. Applying this threshold to observations of gas in nearby galaxies reproduces the observed star formation rate (Lada et al. 2010). Krumholz, Dekel \& McKee (2012) show that a fixed star formation rate per free-fall time of $1 \%$ can also explain the observed star formation rate from gas reservoirs across this range of spatial scales.

These star formation prescriptions fit the data in the literature from pc scales to galactic scales, but rely on different physical mechanisms to control the conversion of gas into stars. Distinguishing which (if any) of these are correct, requires first finding gas reservoirs in which the prescriptions predict substantially different star formation rates, and then testing these predictions at physically-meaningful size scales (e.g. the Toomre length). The subset of data in the literature observed at such scales $(\lesssim \mathrm{kpc})$, are predominantly star forming regions within a few hundred parsecs of the Sun, or observations of gas/stars in disks of nearby (mostly spiral) galaxies. However, the physical properties of the gas in these environments (e.g. density, temperature, velocity dispersion, interstellar radiation field, pressure, cosmic ray ionization rate) are similar, making them a poor choice for distinguishing between the models.

The central $500 \mathrm{pc}$ of the Milky Way - the central molecular zone (CMZ) $\dagger$ - contains $\sim 10 \%$ of the total molecular gas in the Galaxy. The gas conditions in this environment are extreme compared to that in the solar neighborhood and the disks of nearby galaxies: the density, temperature, velocity dispersion, interstellar radiation field, pressure, cosmic ray ionization rate etc. range from factors of a few to several orders of magnitude larger in the CMZ. Lying at a fraction of the distance to nearby galaxies $(\sim 8.4 \mathrm{kpc})$, the CMZ can also be studied in great detail. As such, the CMZ offers a power laboratory for testing the predictions of different physical mechanisms governing the conversion of gas into stars.

Below I attempt to summarize our recent work investigating star formation in this extreme environment. I begin by focusing on whether the star formation is different in the Galactic center compared to the rest of the Galaxy.

\section{Is Star formation different in the Galactic center and the disk of the Milky Way?}

Many independent studies have attempted to answer this question (e.g. Yusef-Zadeh et al. 2009, Immer et al. 2012a, Longmore et al. 2013a). These studies have reached different conclusions about whether the gas in the CMZ is consistent with the predictions of galactic-scale star formation relations, leading to some confusion. The confusion is exacerbated by several factors. Firstly, the studies use different observational methods to infer the gas mass and star formation rate. Each of these methods have different systematic biases, which may potentially influence the conclusions. Secondly, the studies often cover different galactocentric radius $\left(R_{\mathrm{GC}}\right)$ ranges, typically from $\mathrm{R}_{\mathrm{GC}}<150 \mathrm{pc}$ to $\mathrm{R}_{\mathrm{GC}}<500 \mathrm{pc}$, making it difficult to directly compare the reported gas masses and star formation rates. However, after taking this into account, the measured star formation rates and gas masses in the literature are in fact consistent to within a factor of a few. Given the potential systematics, this is reassuring!

Rather than focusing on the differences between the independent measurements, it is instructive to consider what we can learn from the agreement between them.

$\dagger$ As a note of warning, the dimensions of the CMZ are poorly defined, which can lead to some confusion in comparing results from different studies of the region. Various authors refer to the outer galactocentric radius of the CMZ as ranging from $\sim 200 \mathrm{pc}$ to $500 \mathrm{pc}$. 
Un-controversially, all observations show that the CMZ contains roughly $\sim 5-10 \%$ of the total molecular gas in the Galaxy and $\sim 5-10 \%$ of the total star formation rate. It follows trivially that if a star formation relation predicts that the star formation rate depends only on the total amount of gas (e.g. in nearby disk galaxies, where a simple proportionality of the star formation rate to the molecular gas mass is commonplace - Bigiel et al. 2008, Bigiel et al. 2011), then the CMZ fits on the relation with the rest of Galaxy. However, if the predicted star formation relation depends on gas density in any way, the gas in the CMZ and the disk of the Milky Way can not fit on the same relations.

Most of the commonly-used star formation relations have a density-dependence on the predicted star formation rate. So the question of whether or not the gas in the CMZ fits on the same relation as that in the disk boils down to whether there is a difference in gas density between the regions. This is not trivial to answer. While it is straightforward to derive a gas surface density, the fact that we sit in the plane of our own Galaxy makes if difficult to determine the line of sight extent of the gas emission required to convert the column density to a volume density. The inferred volume density then depends on the assumed geometry of the gas. If one assumes the total measured gas mass within $\mathrm{R}_{\mathrm{GC}}<500 \mathrm{pc}$ is distributed uniformly in a disk of that radius and observed scale height, the gas volume density would be $\sim 100 \mathrm{~cm}^{-3}$ - similar to the average volume density of molecular clouds in the disk. In which case there would be no discrepancy between the observed star formation rates in the CMZ and the disk.

Several lines of evidence suggest this geometry is unlikely. Firstly, radiative transfer modeling of molecular line observations show that the gas in the CMZ is on average two orders of magnitude more dense than gas in the disk. Secondly, looking at nearby barred spiral galaxies of the same morphological type as the Milky Way, the gas in the inner few hundred parsecs typically has a very small volume filling factor, and usually forms a circumnuclear gas ring orbiting the galactic center. Indeed, the gas in the inner $100 \mathrm{pc}$ of the Milky Way has been successfully modeled as a circumnuclear ring (Molinari et al. 2011), requiring a low volume filling factor. These lines of evidence suggest that the average gas density in the CMZ is closer to $10^{4} \mathrm{~cm}^{-3}$ (see Longmore et al. 2013a for a detailed discussion). In which case, the gas in the CMZ and disk of the Milky Way can not fit on the same density-dependent star formation relations. Also note that nearly all of the gas in the CMZ lies close to or above the threshold for efficient star formation proposed by Lada et al. (2010) and Heiderman et al. (2010) and yet most of the gas is not forming stars.

Additionally, one can highlight large mass reservoirs within the CMZ which strongly violate the predictions of all star formation relations. The region $1^{\circ}<l<3.5^{\circ},|b|<0.5^{\circ}$ is particular striking in this regard. It contains $10^{7} \mathrm{M}_{\odot}$ of dense molecular gas - enough to form 1000 Orion-like clusters - but the present-day star formation rate within this gas is only equivalent to that in Orion.

We conclude that, "yes", the star formation in the CMZ is different from that in the disk of the Milky Way. In particular, given the large reservoir of dense gas available to form stars, the star formation rate lies one to two orders of magnitude lower than predicted from galactic-scale star formation relations. The fact that $10 \%$ of the gas in our own Galaxy deviates so drastically from these predictions, calls into question the universality of such relations. And if we cannot understand these relations in our own Galaxy (especially the largest reservoir or dense molecular gas therein), how do we extrapolate to external systems with confidence? 


\section{What is causing the difference between the conversion of gas into stars in the Galactic center and the disk?}

The question then becomes, what is suppressing star formation by between one to two orders of magnitude in the CMZ compared to that in the disk? To try and address this question, in Kruijssen et al. (2013a) [hereafter K13] we attempt to quantify the ability of different suppression mechanisms to slow down star formation in the Galactic center environment. We separate the mechanisms into 'global' and 'local' depending on whether they operate on scales larger or smaller than the vertical scale height, respectively.

In terms of the global support mechanisms, for the gas in the $\mathrm{CMZ}$ at $\mathrm{R}_{\mathrm{GC}}>100 \mathrm{pc}$ it is the stars, and not the gas, that dominate the gravitational potential. In order for this gas to form stars, the gas must become self-gravitating. The time scale for this is approximately given by the ratio of the Toomre $\mathrm{Q}$ value for the gas divide by the epicyclic frequency, $\mathrm{Q} / \kappa$, which for the gas in the $\mathrm{CMZ}$ at this radius is $\sim 20 \mathrm{Myr}$. While this certainly contributes to slowing down star formation at $\mathrm{R}_{\mathrm{GC}}>100 \mathrm{pc}$, the gas at $\mathrm{R}_{\mathrm{GC}} \sim 100 \mathrm{pc}$ is close to self-gravitating, so another mechanism is required to suppress star formation there.

Many mechanisms that act on a size scale smaller than the vertical scale height can potentially suppress star formation. Using the most recently available data, K13 consider the effect of galactic tides, turbulence, the IMF, the atomic-molecular phase transition, radiation pressure and cosmic rays. They conclude that turbulent pressure dominates by several orders of magnitude. But this cannot be the full picture because turbulence dissipates on a vertical disk-crossing time, which in the Galactic center environment is very short, $\sim 0.5 \mathrm{Myr}$. Something is required to drive the turbulence.

K13 show that feedback and classical drivers of turbulence (e.g. Mac Low \& Klessen 2004) are not effective: magnetorotational instabilities, gravitational instabilities, protostellar outflows, ionizing radiation and SNe are all several orders of magnitude too small to match the measured turbulent energy dissipation rate. They conclude that energy injection must be coming from larger scales. One potential energy injection mechanism is the kinetic energy of gas that is known to flow from the disk to the center of barred spiral galaxies like the Milky Way. The amount of energy injected depends on the mass inflow rate. Making some simple assumptions about the magnitude of the inflow shows a steady-state inflow rate is still an order of magnitude too low.

An additional mechanism which may help provide the required energy injection is the 'kitchen sink' model of Montenegro et al. (1999). In this scenario, non self-gravitating gas from the disk falls into a stellar mass-dominated potential. This leads to geometric gas convergence, where gas gets crammed to smaller and smaller radii i.e. continued gas flows can only be funneled to ever smaller volumes. The gas gets compressed to high density despite not being self-gravitating. Within this gas, acoustic instabilities drive spiral waves which increase the turbulent pressure. The compression continues until reaching a point where the gas density becomes larger than the stellar density. At which point the gas undergoes gravitational collapse and can form stars.

\subsection{A self-consistent cycle of star formation in the Galactic center}

K13 attempt to explain the above results with a self-consistent cycle of star formation. In this scenario, gas in the disk feels a torque from the stellar bar, loses angular momentum and gets funneled towards the Galactic center. By analogy with nearby barred spiral galaxies, the mass inflow likely occurs along the leading edge of the bar. This gas falls into a stellar mass-dominated potential and so is initially not self-gravitating. Acoustic instabilities of inflowing gas drive a turbulent cascade leading to a highly 
environmentally-dependent density threshold for star formation. The gas mass builds up to the critical density threshold for gravitational instability and then undergoes rapid star formation and gas consumption.

There are several implications of this scenario. Firstly, the conversion of gas into stars is determined by processes at multiple different scales which directly affect each other: the overall gas supply is regulated on global (kpc) scales, while the gas consumption time is set locally (pc scales). Secondly, the rate-limiting factor of star formation is the long evolution of gas towards gravitational collapse. Thirdly, star formation should occur at specific locations within the Galactic center. Specifically, the '100pc ring' may represent the unstable phase of the proposed cycle. Fourthly, the star formation in the Galactic center should be episodic. The chance of observing the region in a starburst vs quiescent phase is determined by the relative timescales of the phases, i.e. by comparing (i) the inflow dynamical time ( 5 to $100 \mathrm{Myr}$ ), (ii) the time for the gas to become unstable against its own self-gravity $(\mathrm{Q} / \kappa \sim 1$ to $20 \mathrm{Myr}$ ), and (iii) the very short gas consumption time scale (e.g. several dynamical times $\sim$ Myr). Finally, in the quiescent phase, the fact that the gas is not self-gravitating may allow it to proceed to the central supermassive black hole without forming stars, suggesting the phases of low star formation activity might be linked to efficient black hole growth.

It is interesting to note that this cycle of star formation is very general, so it may well hold in the centers of other galaxies. In which case, the inferred duty cycle for star formation leads to direct predictions for the relative number of these external galaxies, which should lie above and below the star formation rates expected from galactic star formation relations given the gas reservoir available to form stars.

\section{How similar is the gas in the center of the Milky Way to other star forming environments across cosmological timescales?}

Having concluded that the gas in the center of the Galaxy strongly violates galacticscale star formation relations, and then put forward a scenario to explain what might be causing this, it is pertinent to consider whether this has any bearing at all to star formation in a cosmological context. It may be the case, for example, that the gas in the center of our own Galaxy is pathological or unique for some reason. In which case any conclusions we draw are of limited significance cosmologically-speaking. In other words, the role that the $\mathrm{CMZ}$ can play in our understanding of star formation in a cosmological context depends on how similar the properties of its gas are to other environments across the Universe.

In Kruijssen \& Longmore (2013) we aim to address this by comparing the gas properties in a representative, but not exhaustive, sample of star formation regions across the full range of known environments. This approach is beset with problems: the data sets are heterogeneous, use different observational tracers, and are sensitive to a large range in spatial resolution. Our approach was to try and identify the observational properties that can be most robustly compared between the different samples. In practical terms, this meant we were limited by the most distant sources in the sample (the high-redshift galaxies), for which observations in the literature report robust sizes, velocity dispersions, stellar masses and gas masses. The results of this analysis showed that in terms of baryonic composition, kinematics, and densities, the CMZ gas is indistinguishable from that in high-redshift galaxies and starbursts. 
The conclusion from this work is that, at the level of current observational precision, the CMZ is the nearest high-redshift galaxy analogue. As such, it can play a role in understanding star formation in extreme environments like those in the early Universe.

\section{What can detailed future studies of star formation in the Galactic center tell us?}

The four most massive and dense clouds in the Milky Way are all found at a similar location in the Galaxy. They are part of the dense circumnuclear molecular gas ring orbiting the Galactic center at a radius of $\sim 100 \mathrm{pc}$ (Molinari et al. 2011, Immer et al. 2012b), so all have very similar environmental conditions (Longmore et al. 2012, Longmore et al. 2013b). The supermassive black hole at the center of the Galaxy (Sgr A*) and its surrounding nuclear star cluster are much closer to one side of the ring (Molinari et al. 2011) and there is evidence that they are interacting with gas in the ring (Herrnstein \& Ho 2005). Intriguingly, the four most massive and dense clouds lie just downstream from the ring's pericenter passage with $\mathrm{Sgr} \mathrm{A}^{*}$ and the cloud farthest downstream shows signs of most active star formation. In a recent paper we put forward the idea that the interaction of the ring with the strong gravitational potential at the center of the Galaxy compresses the gas, which loses energy via shocks, triggering star formation (Longmore et al. 2013b). This paper made specific predictions for how this should affect the gas properties. Two independent research groups (Kruijssen, Dale \& Longmore, and Lucas \& Bonnell) have been running numerical simulations of gas orbiting the Galactic center to test these predictions. The initial results of both teams show that the scenario is feasible, and the simulations of Kruijssen, Dale \& Longmore (in prep.) confirm that the model predictions are consistent with the observed gas properties. Comparing the observed gas kinematics with a parameter space study of gas on different orbital trajectories allows us to tightly constrain how long it has been since each of the clouds passed pericenter passage with $\operatorname{Sgr} \mathrm{A}^{*}$ - i.e. the time at which star formation was instigated. For the first time we have a causally-related system for which we know the global environmental properties, the initial conditions of the gas before star formation (the clouds up-stream from pericenter passage with Sgr A*), and have four different snapshots of gas being converted into stars with known absolute time intervals since star formation was instigated. This system provides a truly unique laboratory to directly observe the physics shaping the IMF.

\section{Summary}

The Milky Way contains large reservoirs of gas with properties directly comparable to most of the known range of star formation environments in the Universe. As such, it is an excellent template for studying star formation across cosmological timescales. Future studies of gas in the Milky Way will provide insight into star formation in high-redshift clouds and galaxies and super star cluster formation.

\section{Acknowledgements}

I would like to thank Diederik Kruijssen for comments on the text and many other collaborators - John Bally, Leonardo Testi, Jill Rathborne, Cara Battersby, Eli Bressert, Andrew Walsh, Cormac Purcell, Juergen Ott, Nate Bastian, Sergio Molinari, Joanna Ascenso, Joao Alves, Jim Jackson, Jonathan Foster - who have made working on this so much fun. I would also like to thank the organizers of the symposium for putting together a very stimulating and enjoyable conference. Finally, I am grateful to the Aspen Center 
for Physics for their hospitality and to the National Science Foundation for support, Grant No. 1066293.

\section{References}

Bigiel, F., Leroy, A., Walter, F., Brinks, E., de Blok, W. J. G., Madore, B., \& Thornley M. D. 2008 AJ 136,2846

Bigiel, F., et al. 2011, AJ,730, L13

Bressert, E., Ginsburg, A., Bally, J., Battersby, C., Longmore, S., \& Testi, L. 2012, ApJ 758, 28

Burkert, A. \& Hartman, L. 2013, ApJ 773, 48

Elmegreen, B. G. 1987, ApJ 312, 626

Gutermuth, R. A., Pipher J. L., Megeath, S. T., Myers, P. C., Allen, L. E., \& Allen T. S. 2011, ApJ 739, 84

Heiderman, A., Evans, II N. J., Allen, L. E., Huard, T., \& Heyer, M. 2010, ApJ 723, 1019

Herrnstein, R. M. \& Ho, P. T. P. 2005, ApJ 620, 287

Immer, K., Schuller, F., Omont, A., \& Menten, K. M. 2012, A\&A 537, 121

Immer, K., Menten, K. M., Schuller, F., \& Lis, D. C. 2012, A $\& A$ 548, 120

Kennicutt, Jr. R. C. 1998a, ARA $\& A$ A 36, 189

Kennicutt, Jr. R. C. 1998b, ApJ 498, 541

Krumholz, M. R., Dekel, A., \& McKee, C. F. 2012, ApJ 745, 69

Kruijssen, J. M. D., Longmore, S. N., Elmegreen, B. G., Murray, N., Bally, J., Testi, L., \& Kennicutt, R. C. Jr. 2013, submitted MNRAS arXiv:1303.6286

Kruijssen, J. M. D. \& Longmore, S. N. 2013, MNRAS 435, 2598

Lada, C. J., Lombardi, M., \& Alves, J. F. 2010, ApJ 724, 687

Lada, C. J., Forbrich, J., Lombardi, M., \& Alves, J. F. 2012, ApJ 745, 190

Leroy, A. K., Walter, F., Brinks, E., Bigiel, F., de Blok, W. J. G., Madore, B., \& Thornley, M. D. 2008 AJ 136, 2782

Longmore, S. N., et al. 2012, ApJ 746, 117

Longmore, S. N., et al. 2013a, MNRAS 429, 987

Longmore S. N., et al. 2013b, MNRAS 433, 15

Mac Low, M.-M. \& Klessen, R. S. 2004, Reviews of Modern Physics 76, 125

Molinari, S., et al. 2011, ApJ 735, L33

Montenegro, L. E., Yuan, C., \& Elmegreen, B. G. 1999, ApJ 520, 592

Schmidt, M. 1959, ApJ 129, 243

Silk, J. 1997, ApJ 481, 703

Yusef-Zadeh, F., et al. 2009, ApJ 702, 178 